\title{
Article \\ Prediction of Anticancer Peptides with High Efficacy and Low Toxicity by Hybrid Model Based on 3D Structure of Peptides
}

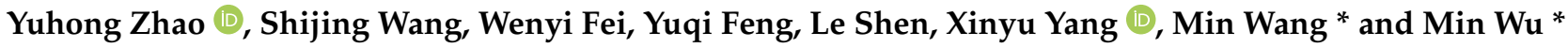 \\ State Key Laboratory of Natural Medicines, School of Life Science and Technology, China Pharmaceutical \\ University, Nanjing 210009, China; zhaoyuhong96@163.com (Y.Z.); wsjwendy@sina.com (S.W.); \\ m15850658512@163.com (W.F.); 3219030694@stu.cpu.edu.cn (Y.F.); sl865070@163.com (L.S.); \\ beiliyashizhu@163.com (X.Y.) \\ * Correspondence: minwang@cpu.edu.cn (M.W.); mickeywu2001@163.com (M.W.)
}

Citation: Zhao, Y.; Wang, S.; Fei, W.; Feng, Y.; Shen, L.; Yang, X.; Wang, M.; $\mathrm{Wu}, \mathrm{M}$. Prediction of Anticancer Peptides with High Efficacy and Low Toxicity by Hybrid Model Based on 3D Structure of Peptides. Int. J. Mol. Sci. 2021, 22, 5630. https://doi.org/ $10.3390 /$ ijms 22115630

Academic Editor: Hanoch Senderowitz

Received: 15 March 2021

Accepted: 19 May 2021

Published: 26 May 2021

Publisher's Note: MDPI stays neutra with regard to jurisdictional claims in published maps and institutional affiliations.

Copyright: (C) 2021 by the authors Licensee MDPI, Basel, Switzerland. This article is an open access article distributed under the terms and conditions of the Creative Commons Attribution (CC BY) license (https:// creativecommons.org/licenses/by/ $4.0 /)$.

\begin{abstract}
Recently, anticancer peptides (ACPs) have emerged as unique and promising therapeutic agents for cancer treatment compared with antibody and small molecule drugs. In addition to experimental methods of ACPs discovery, it is also necessary to develop accurate machine learning models for ACP prediction. In this study, features were extracted from the three-dimensional (3D) structure of peptides to develop the model, compared to most of the previous computational models, which are based on sequence information. In order to develop ACPs with more potency, more selectivity and less toxicity, the model for predicting ACPs, hemolytic peptides and toxic peptides were established by peptides 3D structure separately. Multiple datasets were collected according to whether the peptide sequence was chemically modified. After feature extraction and screening, diverse algorithms were used to build the model. Twelve models with excellent performance (Acc > 90\%) in the ACPs mixed datasets were used to form a hybrid model to predict the candidate ACPs, and then the optimal model of hemolytic peptides (Acc $=73.68 \%$ ) and toxic peptides $(\mathrm{Acc}=85.5 \%)$ was used for safety prediction. Novel ACPs were found by using those models, and five peptides were randomly selected to determine their anticancer activity and toxic side effects in vitro experiments.
\end{abstract}

Keywords: anticancer peptides; hemolytic peptides; toxic peptides; machine learning; ensemble algorithms; hybrid models; three-dimensional structure; multiple datasets

\section{Introduction}

According to the latest cancer statistics, cancer incidence and death rates are increasing year by year [1,2]. Traditional cancer treatment methods mainly include surgery, radiation therapy, chemical drugs and macromolecular targeted drugs. However, cancer treatment continues to face the challenge of increasing resistance to chemical and receptor-targeted anticancer drugs. Researchers identified a class of bioactive peptides with antitumor activity, named anticancer peptides (ACPs), which are found in a wide range of organisms, including mammals, amphibians, insects, plants and microorganisms [3]. As a new potential drug for cancer treatment, ACPs are cationic amphiphilic peptides with a length of about $5-50$ amino acids, which are characterized by a simple structure, easily synthesized, easily modified chemically and less immunogenic [4]. Owing to the increased proportion of phosphatidylserine (negatively charged) on the surface of cancer cells compared to normal cells, cationic amphiphilic peptides may be an effective and highly selective antitumor drug. The antitumor mechanisms of ACPs can be divided into two types: selective membrane destruction and non-membrane dissolution, which include inhibition of angiogenesis and promotion of tumor cell apoptosis [5]. Despite these advantages, ACPs still face challenges before becoming effective clinical agents, such as poor stability, hemolysis and toxicity to normal tissue cells. The stability of peptides can be improved on various ways, including incorporation of unnatural amino acids, cyclization and modification of 
the chemical skeleton [6]. Therefore, it is essential to develop methods to identify safer and more effective ACPs.

Machine learning derived from artificial intelligence and statistics is one of the key research directions in the field of data analysis at present. The application of machine learning algorithms in drug research and development greatly speeds up the process of early drug screening. Basic machine learning algorithms includes naive Bayes (NB), support vector machine (SVM), random forests (RF), K nearest neighbor (KNN), artificial neural networks (ANNs), ensemble algorithms, etc. Due to identifying potential novel ACPs using experimental methods require considerable time and expense, in order to aid wet-laboratory researchers discover novel ACPs, various machine learning approaches are used to ACPs recognition [7].

In the past decades, there have been many significant studies on the prediction of ACPs based on sequence features. In the beginning, Tyagi et al. [8] used the SVM method to construct an online predictor AntiCP based on amino acid composition, dipeptide composition and binary profile pattern. Then, Hajisharififi et al. [9] developed a model of SVM based on Chou's pseudo-amino acid composition (PseAAC) and local alignment kernel. After that, researchers have established many predictors, for example, ACPP [10], iACP [11], MLACP [12], iACP-GAEnsC [13], ACPred-FL [14], SAP [15], TargetACP [16], ACPred [17], mACPpred [18], ACPred-Fuse [19], PTPD [20], ACP-DL [21], PEPred-suite [22], AntiCP 2.0 [23], CancerGram [24], DeepACP [25] and ENNAACT [26], most of them adopt diverse feature extraction methods combined with various machine learning algorithms. All of the above predictors performed well in distinguishing between ACPs and non-ACPs. Additionally, in order to screen safe candidate peptide drugs, some prediction models of toxic peptide and hemolytic peptide have been developed successively [27-33].

Although tremendous advances in the field of ACPs prediction, these approaches are almost entirely based on peptide sequences of feature extraction and use very similar datasets. In addition to the basic characteristics of peptide sequence, the 3D structure of ACPs plays a key role in inhibiting tumor cell proliferation. SATPdb [34] is a database annotating the tertiary structure of various therapeutic peptides using PEstrMOD [35], homology modeling [36] and I-TASSER Suite [37] methods, which provides a data basis of structure-based activity and toxicity prediction of ACPs. Consequently, in this study, we collected five datasets of 3D structure of ACPs and used a variety of machine learning algorithms to establish the model. Similarly, the tertiary structure datasets of hemolytic peptides and toxic peptides were collected to construct models for predicting the safety of ACPs. Then, 12 algorithms with the better performance were selected from the two ACPs datasets to form a mixed model to screen ACPs from antimicrobial peptides (AMPs). The optimal prediction models of hemolytic peptides and toxic peptides were used to ensure the safety of candidate ACPs (Figure 1). To our knowledge, this is the first reported method to simultaneously predict ACPs activity, hemolysis and toxicity based on the 3D structure of peptides. Novel ACPs were discovered by the above methods, and their anticancer efficacy and toxicity were evaluated by in vitro experiments. 


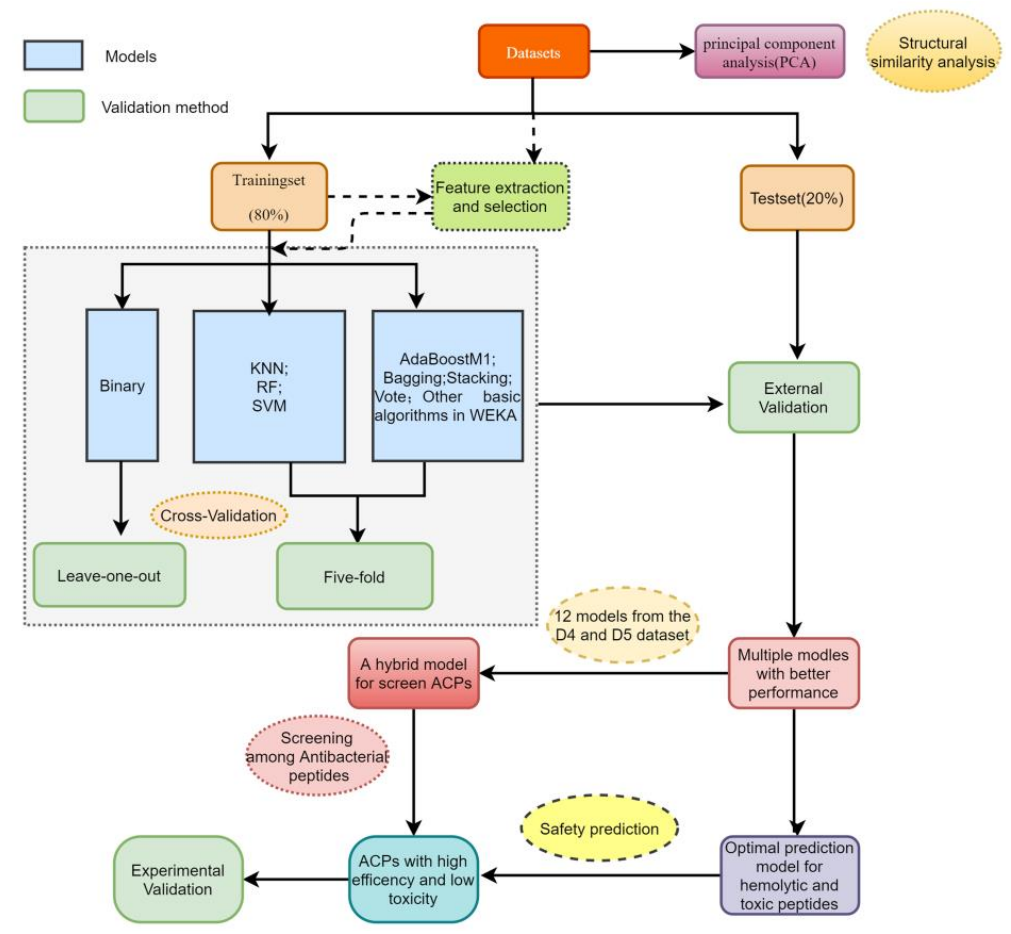

Figure 1. The flowchart describes the overall implementation approach for screening high-efficiency and low-toxicity ACPs.

\section{Results}

\subsection{The Composition of Multiple Datasets}

The positive and negative sample compositions of five ACPs datasets, three hemolytic peptides datasets and three toxic peptides datasets are shown in Figure 2A-C. Figure 2D shows the training set and test set division of all datasets. The above datasets are detailed in Section 4.1 Datasets.

A

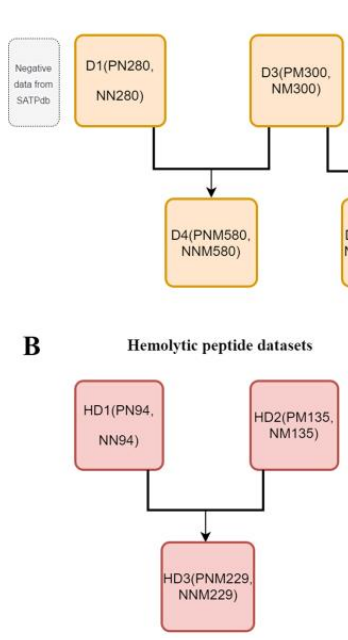

D

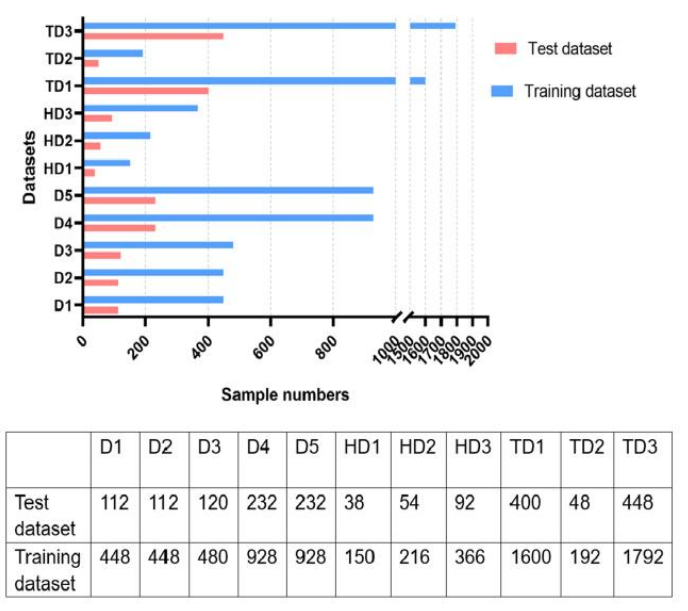

Figure 2. Composition of multiple datasets. (A) Number of positive and negative samples in five datasets of anticancer peptides. PN, NN, NNSW, PM and NM indicate positive natural peptides, negative natural peptides, negative natural peptides derived from SwissProt, positive chemically modified peptides and negative chemically modified peptides, respectively. PNM represents a positive dataset of mixed natural peptides and chemically modified peptides. NNM implies a negative dataset of mixed natural peptides and chemically modified peptides. (B) The number distribution of positive and negative samples of three hemolytic peptides datasets. $(C)$ The number distribution of positive and negative samples in three toxic peptide datasets. (D) Data splitting of all datasets. Blue indicates training set; pink indicates test set. 


\subsection{Structural Similarity Analysis}

The first three principal components were extracted from structural features for similarity analysis. The positive data and negative data of the ACPs datasets were obviously distinguished in the three-dimensional space. The concentrated distribution of polypeptides with anticancer activity indicated that the extracted features were very effective. In particular, the chemically modified peptide dataset D3 performed better than other ACPs datasets. The distinction between positive and negative data was not obvious in the hemolytic peptide datasets, and the data distribution was relatively less concentrated. Toxic peptides have very similar features and can clearly distinguish between positive and negative data. Structural similarity analysis of mixed peptide datasets is shown in Figure 3, and other datasets in Figure S1.

A

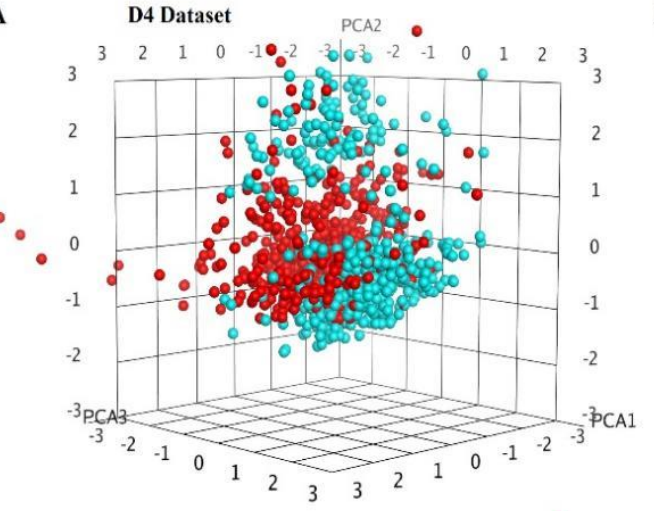

C

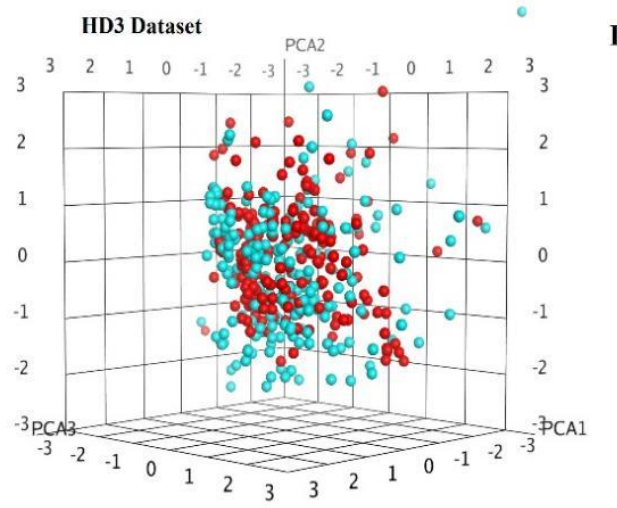

B

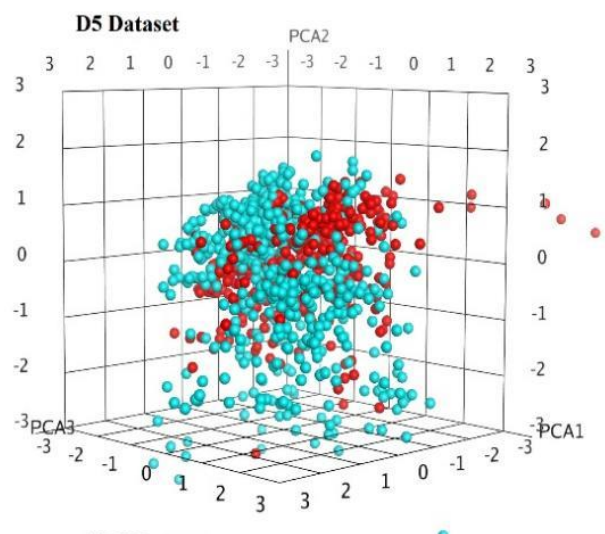

D

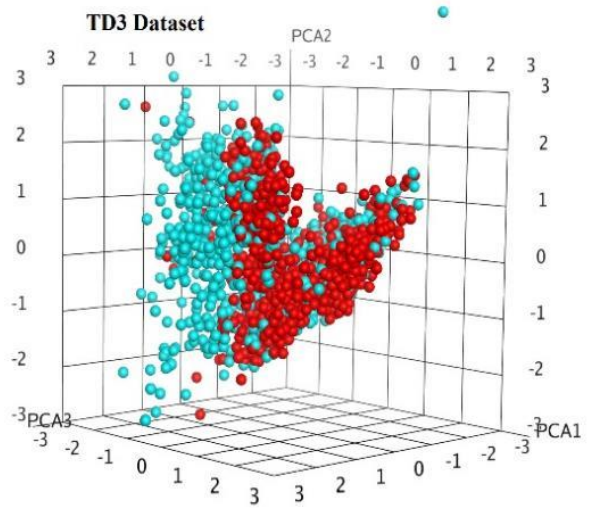

Figure 3. Structural similarity analysis based on principal component analysis. (A) ACPs mixed dataset D4. (B) ACPs mixed dataset D5 (data of negative natural peptides were obtained from Swissprot). (C) Hemolytic peptide mixed dataset HD3. (D) Toxic peptide mixed dataset TD3. Blue indicates negative data and red indicates positive data.

\subsection{Development of Models on ACPs Dataset}

In this study, five ACP datasets were collected to explore the influence of different datasets on model construction. The model of D2 performs better than the model of D1 in the natural peptide dataset (Table 1, Figure 4A), which indicates that ACPs and AMPs have similar structures, and their differentiation is slightly weaker than that of ACPs and peptides derived from Swissprot. The model performance of chemically modified peptide dataset D3 was better than that of natural peptide dataset D1 and D2, illustrating that the feature extraction method in this study was easier to extract the chemically modified features of peptides, so the chemically modified ACPs were easier to distinguish from modified AMPs. Similarly, the model of mixed peptide dataset D5 was superior to the model of D4 (Table 2). Overall, it is crucial to select the appropriate dataset when constructing the prediction model. 
Table 1. In the ACPs datasets D1, D2 and D3, excellent models were constructed based on multiple algorithms and different features.

\begin{tabular}{|c|c|c|c|c|c|c|c|c|c|c|}
\hline \multirow[b]{2}{*}{ Dataset } & \multirow[b]{2}{*}{ Method } & \multirow[b]{2}{*}{$\begin{array}{l}\text { Number of } \\
\text { Features }\end{array}$} & \multicolumn{4}{|c|}{ Training Dataset } & \multicolumn{4}{|c|}{ Test Dataset } \\
\hline & & & $\begin{array}{l}\text { Sen } \\
(\%)\end{array}$ & $\begin{array}{l}\text { Spc } \\
(\%)\end{array}$ & $\begin{array}{l}\text { Acc } \\
(\%)\end{array}$ & MCC & $\begin{array}{l}\text { Sen } \\
(\%)\end{array}$ & $\begin{array}{l}\text { Spc } \\
(\%)\end{array}$ & $\begin{array}{l}\text { Acc } \\
(\%)\end{array}$ & MCC \\
\hline D1 & Vote & 306 & 90.87 & 97.38 & 94.20 & 0.89 & 96.72 & 96.08 & 96.43 & 0.93 \\
\hline D1 & KNN (k = 9) & 24 & 81.55 & 91.69 & 86.59 & 0.74 & 72.13 & 92.16 & 81.25 & 0.65 \\
\hline D1 & Bagging (J48) & 24 & 83.56 & 87.77 & 85.71 & 0.71 & 95.08 & 94.12 & 94.64 & 0.89 \\
\hline D2 & RF & 318 & 78.10 & 97.33 & 89.48 & 0.79 & 96.43 & 98.21 & 97.32 & 0.95 \\
\hline D2 & $\begin{array}{l}\text { AdaBoostM1 } \\
\text { (J48) }\end{array}$ & 318 & 92.41 & 93.27 & 92.84 & 0.86 & 96.43 & 98.21 & 97.32 & 0.95 \\
\hline D2 & RF & 148 & 83.02 & 94.22 & 88.80 & 0.77 & 96.43 & 94.64 & 95.54 & 0.91 \\
\hline D2 & $\begin{array}{l}\text { Bagging } \\
\text { (SMO) }\end{array}$ & 148 & 91.52 & 90.13 & 90.83 & 0.82 & 96.43 & 96.43 & 96.43 & 0.93 \\
\hline D2 & KNN & 20 & 92.37 & 90.58 & 91.50 & 0.83 & 96.43 & 96.43 & 96.43 & 0.93 \\
\hline D2 & $\begin{array}{c}\text { AdaBoostM1 } \\
\text { (J48) }\end{array}$ & 20 & 92.41 & 93.27 & 92.84 & 0.86 & 96.43 & 98.21 & 97.32 & 0.95 \\
\hline D3 & Bagging (IBK) & 332 & 97.50 & 97.92 & 97.71 & 0.95 & 100.00 & 98.33 & 99.17 & 0.98 \\
\hline D3 & KNN $(\mathrm{k}=9)$ & 37 & 98.38 & 97.95 & 98.13 & 0.96 & 100.00 & 100.00 & 100.00 & 1 \\
\hline D3 & $\begin{array}{l}\text { Bagging } \\
\text { (SMO) }\end{array}$ & 37 & 96.25 & 98.75 & 97.50 & 0.95 & 100.00 & 98.33 & 99.17 & 0.98 \\
\hline D3 & KNN (k = 9) & 13 & 96.04 & 98.79 & 97.50 & 0.95 & 100.00 & 100.00 & 100.00 & 1 \\
\hline D3 & $\begin{array}{l}\text { AdaBoostM1 } \\
\text { (SMO) }\end{array}$ & 13 & 96.69 & 98.31 & 97.49 & 0.95 & 100.00 & 98.33 & 99.17 & 0.98 \\
\hline
\end{tabular}

A
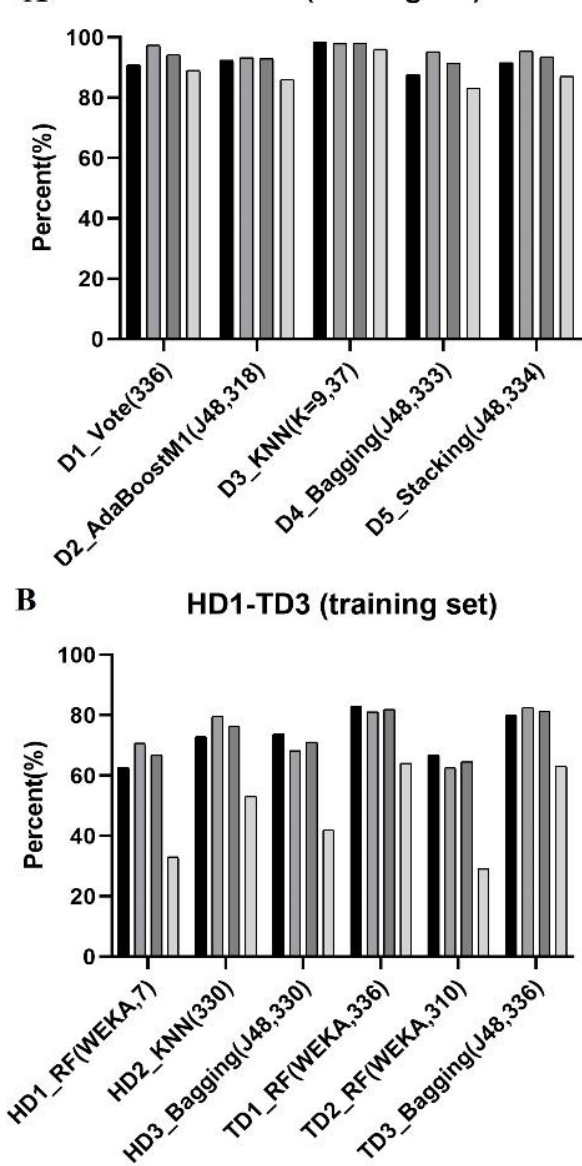

D1-D5 (test set)
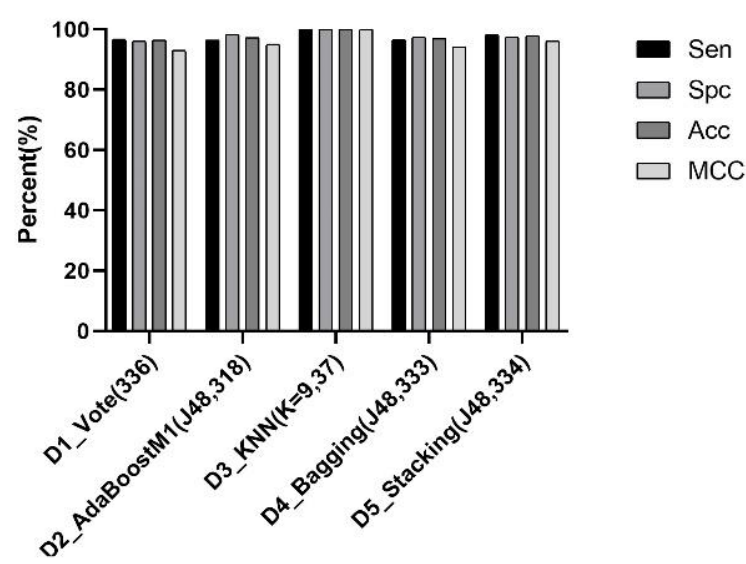

HD1-TD3(test set)
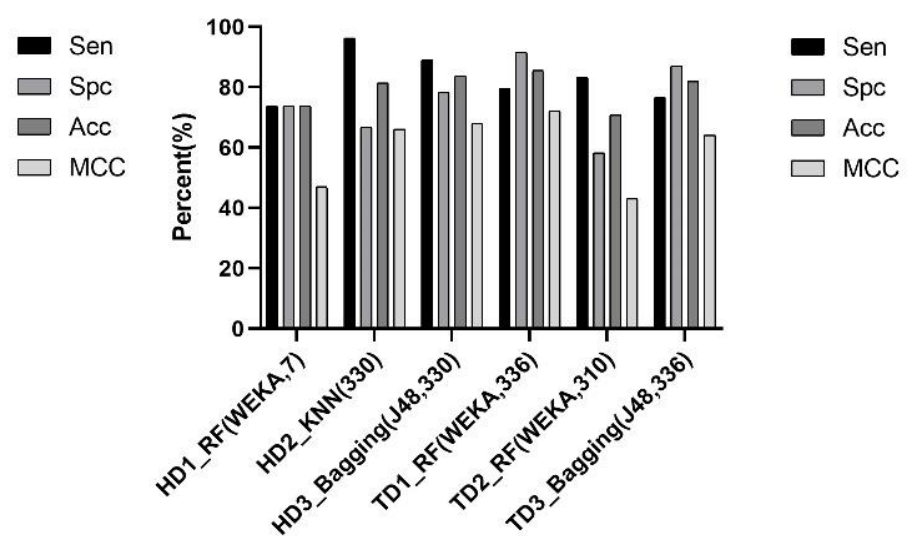

Figure 4. The optimal model for each dataset. (A) The performance of the optimal models of 5 ACPs datasets on the training set and the test set. (B) Performance of optimal models of hemolytic peptide and toxic peptide datasets on training sets and test sets. 
Table 2. In the mixed datasets D4 and D5 of ACPs, excellent models were developed based on a variety of algorithms and different features.

\begin{tabular}{|c|c|c|c|c|c|c|c|c|c|c|}
\hline \multirow[b]{2}{*}{ Dataset } & \multirow[b]{2}{*}{ Method } & \multirow{2}{*}{$\begin{array}{c}\text { Number of } \\
\text { Features }\end{array}$} & \multicolumn{4}{|c|}{ Training Dataset } & \multicolumn{4}{|c|}{ Test Dataset } \\
\hline & & & $\begin{array}{l}\text { Sen } \\
(\%)\end{array}$ & $\begin{array}{l}\text { Spc } \\
(\%)\end{array}$ & $\begin{array}{l}\text { Acc } \\
(\%)\end{array}$ & MCC & $\begin{array}{l}\text { Sen } \\
(\%)\end{array}$ & $\begin{array}{l}\text { Spc } \\
(\%)\end{array}$ & $\begin{array}{l}\text { Acc } \\
(\%)\end{array}$ & MCC \\
\hline D4 & KNN & 333 & 86.36 & 94.10 & 90.30 & 0.81 & 96.55 & 92.24 & 94.40 & 0.89 \\
\hline D4 & $\begin{array}{l}\text { Bagging } \\
\text { (J48) }\end{array}$ & 333 & 87.72 & 95.26 & 91.49 & 0.83 & 96.55 & 97.41 & 96.98 & 0.94 \\
\hline D4 & Binary & 9 & 70.26 & 92.67 & 81.47 & 0.65 & 96.55 & 93.10 & 94.83 & 0.90 \\
\hline D4 & $\begin{array}{l}\text { Stacking } \\
(\mathrm{J} 48)\end{array}$ & 9 & 85.13 & 94.18 & 89.66 & 0.80 & 98.28 & 95.69 & 96.98 & 0.94 \\
\hline D4 & KNN (k = 9) & 14 & 87.26 & 94.77 & 91.05 & 0.82 & 96.55 & 88.79 & 92.67 & 0.86 \\
\hline D4 & $\begin{array}{l}\text { Bagging } \\
\text { (J48) }\end{array}$ & 14 & 87.50 & 93.97 & 90.73 & 0.82 & 97.41 & 96.55 & 96.98 & 0.94 \\
\hline D5 & KNN & 334 & 92.00 & 93.98 & 93.00 & 0.86 & 98.28 & 96.55 & 97.41 & 0.95 \\
\hline D5 & $\begin{array}{l}\text { Stacking } \\
\text { (J48) }\end{array}$ & 334 & 91.59 & 95.47 & 93.53 & 0.87 & 98.28 & 97.41 & 97.84 & 0.96 \\
\hline D5 & KNN (k = 9) & 19 & 89.60 & 93.03 & 91.28 & 0.83 & 99.14 & 94.83 & 96.98 & 0.94 \\
\hline D5 & $\begin{array}{l}\text { Bagging } \\
\text { (REPtree) }\end{array}$ & 19 & 87.50 & 92.46 & 89.98 & 0.80 & 98.28 & 98.28 & 98.28 & 0.97 \\
\hline D5 & $\mathrm{KNN}(\mathrm{k}=9)$ & 17 & 88.40 & 95.08 & 91.70 & 0.84 & 97.41 & 92.24 & 94.83 & 0.90 \\
\hline D5 & Vote & 17 & 87.50 & 95.47 & 91.49 & 0.83 & 97.41 & 96.55 & 96.98 & 0.94 \\
\hline
\end{tabular}

\subsubsection{D1 Dataset}

A total of 306 descriptors were obtained by preliminary feature screening in D1 dataset, and 24 descriptors were obtained by further feature selection method in WEKA (Table S1). A variety of algorithms were selected for model construction based on 306 and 24 features respectively. Then, the optimal model is selected from the classical algorithms Binary, RF, SVM and KNN. From WEKA ensemble algorithm (AdaBoostM1, Bagging, Stacking and Vote) select the optimal model. In the D1 dataset, the best performing model was the Vote algorithm based on 306 features, with accuracy of $94.2 \%$ and MCC of 0.89 in the training set and accuracy of $96.43 \%$ and MCC of 0.93 in the test set.

\subsubsection{D2 Dataset}

A total of 318 descriptors were obtained by preliminary screening in D2 dataset, and 20 descriptors were obtained by further feature screening in WEKA (Table S3). In addition, the dataset has 148 descriptors recommended by the MOE descriptor calculation module (Table S2). Same as the D1 dataset, 6 models with better performance were obtained after model construction and selection. The two models with the best performance are AdaBoostM1 algorithm with J48 primary learner, and the selected features are 318 and 20 , respectively. It achieved accuracy of $92.84 \%$ and MCC of 0.86 on the training set, and accuracy of $97.32 \%$ and MCC of 0.95 on the test set.

\subsubsection{D3 Dataset}

A total of 332 features were selected from the chemically modified peptide dataset D3 after preliminary screening, and the remaining 13 features were further screened by WEKA (Table S5). Furthermore, the dataset has 37 suggested descriptors in the MOE (Table S4). After model construction and selection, five models with excellent performance were obtained. Among them, the model with the best performance is the classical algorithm KNN ( $(k=9)$ based on 37 features. It achieved the highest accuracy of $100 \%$, MCC of 1 on the test dataset and accuracy of $98.13 \%$, MCC of 0.96 on the training dataset.

\subsubsection{D4 Dataset}

A total of 333 descriptors were selected from the mixed peptide dataset D4, and 14 descriptors were obtained by further feature selection by WEKA (Table S7). In addition, the dataset has nine suggested descriptors in the MOE (Table S6). Six models with excellent performance were obtained through model construction and selection. The Bagging 
algorithm based on 333 features with $\mathrm{J} 48$ as the primary classifier has the best performance. It achieved accuracy of $91.49 \%$ and MCC of 0.83 on the training set, and accuracy of $96.98 \%$ and MCC of 0.94 on the test set (Table 2, Figure 4A).

\subsubsection{D5 Dataset}

In the mixed dataset D5, 334 features were selected preliminarily and 17 features were further selected in WEKA (Table S9). Moreover, there were 19 suggested descriptors in the MOE (Table S8). Six models with better performance were obtained through model development and screening. The optimal model was based on 334 descriptors developed using J48 as a secondary classifier of Stacking algorithm, with an accuracy of $93.53 \%$ and MCC of 0.87 (training set), and accuracy of $96.98 \%$ and MCC of 0.94 (test set) (Table 2, Figure $4 \mathrm{~A})$.

Selecting low-dimensional features in each dataset performed as well as models constructed with high-dimensional features. These results suggest that high quality models can be developed as long as there are key features. On D1, D2, D4 and D5 data sets, the performance of the ensemble algorithm was relatively slightly better than that of the classical algorithm. In the five datasets of ACPs, all the models screened above showed excellent internal stability and external predictability.

\subsection{Models Developed on Hemolytic Peptide Dataset}

In the natural dataset HD1 of hemolytic peptide, 308 descriptors were selected preliminarily, and the remaining 7 descriptors were further selected by WEKA (Table S10). The optimal model was the RF algorithm derived from WEKA based on 7 descriptors, with an accuracy of $66.67 \%$ and MCC of 0.33 (training set) and accuracy of $73.68 \%$ and MCC of 0.47 (test set). In the chemically modified peptide dataset HD2, there were 330 features after initial screening, and 18 features were further screened by WEKA (Table S11). The model with the best performance is the KNN algorithm based on 330 features. It achieved accuracy of $76.38 \%$ and MCC of 0.53 on the training set, and accuracy of $81.48 \%$ and MCC of 0.66 on the test set (Table 3, Figure 4B). In the mixed peptide data set HD3, 330 features were screened for the first time and 23 features were further screened by WEKA (Table S12). The optimal model is the Bagging algorithm based on 330 descriptors with J48 as the primary learner, with an accuracy of $71.04 \%$ and MCC of 0.42 (training set), and accuracy of $83.7 \%$ and MCC of 0.68 (test set). In the hemolytic peptide dataset, the model chemically modified peptides developed based on the 3D structure features are superior to the natural peptides. The ensemble algorithm derived from WEKA performed better than the classical algorithm. The optimal models in the three hemolytic peptide datasets had good fit in the training set and good generalization ability in the test set.

Table 3. In the hemolytic peptide dataset HD1, HD2 and HD3, the better performance model is developed according to various algorithms and different features.

\begin{tabular}{|c|c|c|c|c|c|c|c|c|c|c|}
\hline \multirow[b]{2}{*}{ Dataset } & \multirow[b]{2}{*}{ Method } & \multirow[b]{2}{*}{$\begin{array}{l}\text { Number of } \\
\text { Features }\end{array}$} & \multicolumn{4}{|c|}{ Training Dataset } & \multicolumn{4}{|c|}{ Test Dataset } \\
\hline & & & $\begin{array}{l}\text { Sen } \\
(\%)\end{array}$ & $\begin{array}{l}\text { Spc } \\
(\%)\end{array}$ & $\begin{array}{l}\text { Acc } \\
(\%)\end{array}$ & MCC & $\begin{array}{l}\text { Sen } \\
(\%)\end{array}$ & $\begin{array}{l}\text { Spc } \\
(\%)\end{array}$ & $\begin{array}{l}\text { Acc } \\
(\%)\end{array}$ & MCC \\
\hline HD1 & KNN (k=9) & 308 & 67.12 & 61.52 & 64.00 & 0.29 & 73.68 & 57.89 & 65.79 & 0.32 \\
\hline HD1 & Vote & 308 & 81.33 & 80.00 & 80.67 & 0.61 & 73.68 & 57.89 & 65.79 & 0.32 \\
\hline HD1 & Binary & 7 & 78.67 & 76.00 & 77.33 & 0.55 & 63.16 & 57.89 & 60.53 & 0.21 \\
\hline HD1 & RF (WEKA) & 7 & 62.67 & 70.67 & 66.67 & 0.33 & 73.68 & 73.68 & 73.68 & 0.47 \\
\hline HD2 & KNN & 330 & 72.97 & 79.60 & 76.38 & 0.53 & 96.29 & 66.67 & 81.48 & 0.66 \\
\hline HD2 & Vote & 330 & 95.37 & 93.52 & 94.44 & 0.89 & 77.78 & 81.48 & 79.63 & 0.59 \\
\hline HD2 & $\mathrm{RF}$ & 18 & 76.11 & 65.85 & 70.35 & 0.42 & 88.89 & 62.96 & 75.93 & 0.54 \\
\hline HD2 & Vote & 18 & 78.70 & 60.19 & 69.44 & 0.40 & 77.78 & 81.48 & 79.63 & 0.59 \\
\hline HD3 & $\begin{array}{l}\text { Bagging } \\
\text { (J48) }\end{array}$ & 330 & 73.77 & 68.31 & 71.04 & 0.42 & 89.13 & 78.26 & 83.70 & 0.68 \\
\hline HD3 & Binary & 23 & 73.22 & 79.23 & 76.23 & 0.53 & 58.70 & 73.91 & 66.30 & 0.33 \\
\hline HD3 & $\begin{array}{l}\text { Bagging } \\
\text { (RF) }\end{array}$ & 23 & 72.68 & 72.13 & 72.40 & 0.45 & 89.13 & 71.74 & 80.43 & 0.62 \\
\hline
\end{tabular}




\subsection{Models Developed on Toxic Peptide Dataset}

In the natural toxic peptide dataset TD1, there were 336 features after preliminary screening and 22 features were further selected by WEKA (Table S13). The best performing model was the RF algorithm derived from WEKA based on 336 features, with accuracy of $81.94 \%$ and MCC of 0.64 in the training set and accuracy of $85.5 \%$ and MCC of 0.72 in the test set (Table 4, Figure 4B). In the chemically modified peptide dataset TD2, 310 descriptors were selected for the first time, and the remaining 15 descriptors were further screened by WEKA (Table S14). The optimal model was the RF algorithm derived from WEKA based on the 310 features. It achieved an accuracy of $64.58 \%$ and MCC of 0.29 on the training set and an accuracy of $70.83 \%$ and MCC of 0.43 on the test set. In the mixed peptide dataset TD3, 336 descriptors were obtained by preliminary screening and 27 descriptors were obtained by further selecting by WEKA (Table S15). The optimal model was the Bagging algorithm based on 336 features with J48 as the primary learner, with an accuracy of $81.31 \%$ and MCC of 0.63 (training set), and accuracy of $81.92 \%$ and MCC of 0.64 (test set). In the toxic peptide dataset, the performance of the model developed by the features extracted from chemically modified peptides was weaker than that of natural peptides, possibly due to similar chemical modification structures on toxic peptides and AMPs. Additionally, the model developed by the ensemble algorithm is superior to the classical algorithm. The three optimal models have good internal fitting and external predictability.

Table 4. In the toxic peptide dataset TD1, TD2 and TD3, the better performance model was established according to different algorithms and different features.

\begin{tabular}{|c|c|c|c|c|c|c|c|c|c|c|}
\hline \multirow[b]{2}{*}{ Dataset } & \multirow[b]{2}{*}{ Method } & \multirow{2}{*}{$\begin{array}{l}\text { Number of } \\
\text { Features }\end{array}$} & \multicolumn{4}{|c|}{ Training Dataset } & \multicolumn{4}{|c|}{ Test Dataset } \\
\hline & & & $\begin{array}{l}\text { Sen } \\
(\%)\end{array}$ & $\begin{array}{l}\text { Spc } \\
(\%)\end{array}$ & $\begin{array}{l}\text { Acc } \\
(\%)\end{array}$ & MCC & $\begin{array}{l}\text { Sen } \\
(\%)\end{array}$ & $\begin{array}{l}\text { Spc } \\
(\%)\end{array}$ & $\begin{array}{l}\text { Acc } \\
(\%)\end{array}$ & MCC \\
\hline TD1 & KNN & 336 & 81.89 & 80.50 & 81.19 & 0.62 & 80.00 & 77.50 & 78.75 & 0.58 \\
\hline TD1 & RF (WEKA) & 336 & 82.88 & 81.00 & 81.94 & 0.64 & 79.50 & 91.50 & 85.50 & 0.72 \\
\hline TD1 & KNN $(\mathrm{k}=9)$ & 22 & 84.18 & 77.57 & 80.88 & 0.62 & 75.00 & 85.00 & 80.00 & 0.60 \\
\hline TD1 & $\begin{array}{c}\text { Bagging } \\
\text { (J48) }\end{array}$ & 22 & 82.38 & 82.75 & 82.56 & 0.65 & 79.00 & 89.50 & 84.25 & 0.69 \\
\hline TD2 & $\mathrm{KNN}(\mathrm{k}=9)$ & 310 & 74.02 & 59.30 & 66.63 & 0.34 & 45.83 & 83.33 & 64.58 & 0.31 \\
\hline TD2 & RF (WEKA) & 310 & 66.67 & 62.50 & 64.58 & 0.29 & 83.33 & 58.33 & 70.83 & 0.43 \\
\hline TD2 & KNN $(\mathrm{k}=9)$ & 15 & 71.13 & 60.13 & 65.11 & 0.32 & 45.83 & 75.00 & 60.42 & 0.22 \\
\hline TD3 & $\mathrm{KNN}(\mathrm{k}=9)$ & 336 & 80.00 & 79.51 & 79.74 & 0.60 & 71.30 & 75.11 & 73.21 & 0.46 \\
\hline TD3 & $\begin{array}{c}\text { Bagging } \\
\text { (J48) }\end{array}$ & 336 & 80.04 & 82.57 & 81.31 & 0.63 & 76.68 & 87.11 & 81.92 & 0.64 \\
\hline TD3 & $\begin{array}{l}\text { Stacking } \\
\text { (J48) }\end{array}$ & 27 & 76.37 & 82.23 & 79.30 & 0.59 & 74.89 & 87.56 & 81.25 & 0.63 \\
\hline
\end{tabular}

\subsection{Screening of Candidate ACPs}

A total of 1294 AMPs were collected as a dataset to screen peptides with anticancer activity. Since the dataset included chemically modified peptides and natural peptides, we used the models in the ACPs mixed dataset D4 and D5 for ACPs prediction. As shown in Table 2, the 12 models developed in the D4 and D5 data sets were all excellent. In order to increase the accuracy of the prediction model, we tried to combine the prediction results of the 12 models to select ACPs. The screening criterion of the mixed model was that the ACPs were determined only when the predicted results of more than nine (including 9) models were positive. A total of 83 candidate ACPs were screened by the above method. Due to almost all of the 83 anticancer peptides being natural peptides, the optimal model of hemolytic peptide HD1 and toxic peptide TD1 was selected for safety prediction. In the end, we obtained 41 candidate ACPs whose hemolysis and toxicity were predicted to be negative. A total of 5 peptides were randomly selected from 41 candidate anticancer peptides for experimental verification. The sequence and structural information of the five polypeptides are shown in Table 5 and Figure 5, and the information of 41 candidate ACPs is listed in Table S16. 
Table 5. Sequence information of 5 peptides.

\begin{tabular}{ccc}
\hline SATPdb ID & Name & Sequence \\
\hline 16563 & RANATUERIN-2Lb & GILSSIKGVAKGVAKNVAAQLLDTLKCKITGC \\
19566 & Brevinin-2DYd & GIFDVVKGVLKGVGKNVAGSLLEQLKCKLSGGC \\
22121 & Odorranain-C1 & GVLGAVKDLLIGAGKSAAQSVLKTLSCKLSNDC \\
22355 & RANATUERIN2 & GLFLDTLKGAAKDVAGKLEGLKCKITGCKLP \\
27843 & Brevinin-2DYb & GLFDVVKGVLKGAGKNVAGSLLEQLKCKLSGGC \\
\hline
\end{tabular}
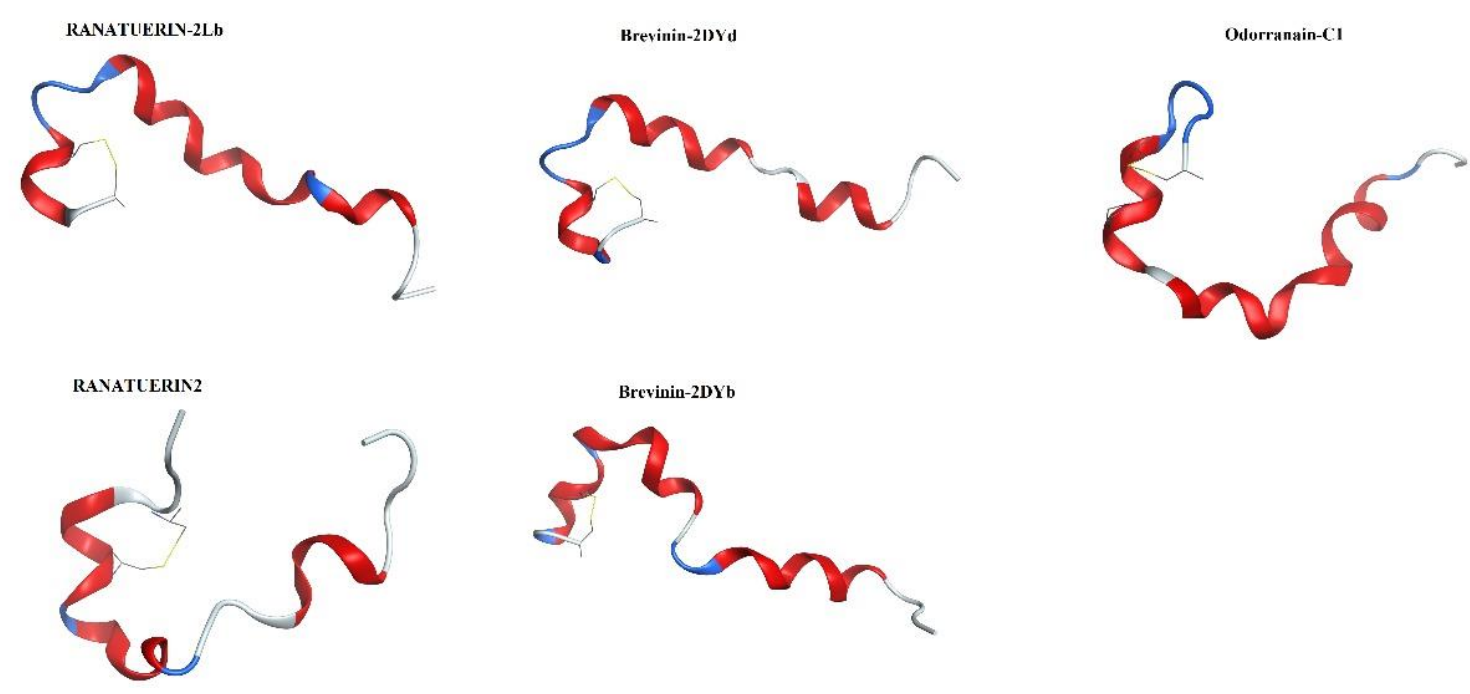

Figure 5. Three-dimensional structure of 5 candidate ACPs.

\subsection{Experimental Verification}

The inhibition rates of several cancer cell lines in the CancerPPD were analyzed statistically [38], with median activity (EC/IC/LC50 $(\mu \mathrm{M}))$ ranging from $17 \pm 3 \mu \mathrm{M}$ to $53 \pm 9 \mu \mathrm{M}$. Therefore, compared with the ACPs in cancerPPD, the candidate five peptides showed anticancer activity when the IC50 value was less than $50 \mu \mathrm{M}$. The anticancer action of the five purified peptides was tested on A549, MCF7, HeLa and LoVo cancer lines, and the concentration that inhibits half of the cell growth was calculated (half-inhibitory concentration [IC50]). Four of the five peptides exhibited anticancer activity and inhibited the growth of at least two types of cancer cells (Figure 6A,B,E, Table 6), which demonstrated the efficacy of the hybrid ACPs model to a certain extent. All the four peptides (Ranatuerin2Lb, Brevinin-2DYd, Odorranain- $\mathrm{C} 1$ and Brevinin-2DYb) had effective killing effects on lung cancer cell A549, especially the IC50 of Brevinin-2DYd and Ranatuerin-2Lb were $2.975 \mu \mathrm{M}$ and $15.32 \mu \mathrm{M}$, respectively. Brevinin-2DYd showed significant inhibitory effects on four cancer cells relative to other peptides.

Table 6. The IC50 values of 5 peptides inhibited the proliferation of 5 kinds of cells in vitro.

\begin{tabular}{cccccc}
\hline IC50 $(\boldsymbol{\mu M})$ & $\begin{array}{c}\text { RANATUERIN-2Lb } \\
\mathbf{( 1 6 5 6 3 )}\end{array}$ & $\begin{array}{c}\text { Brevinin-2DYd } \\
\mathbf{( 1 9 5 6 6 )}\end{array}$ & $\begin{array}{c}\text { Odorranain-C1 } \\
\mathbf{( 2 2 1 2 1 )}\end{array}$ & $\begin{array}{c}\text { Brevinin-2DYb } \\
\mathbf{( 2 7 8 4 3 )}\end{array}$ & $\begin{array}{c}\text { RANATUERIN2 } \\
\mathbf{( 2 2 3 5 5 )}\end{array}$ \\
\hline A549 & 15.32 & 2.975 & 27.31 & 24.01 & $>128$ \\
MCF7 & 45.25 & 25.74 & 41.21 & 37.84 & $>128$ \\
HeLa & 37.23 & 19.69 & 52.83 & 23.26 & $>128$ \\
LoVo & 59.78 & 8.05 & 55.22 & 35.05 & 128 \\
293T & 63.16 & 5.832 & 48.7 & 50.66 & $>128$ \\
\hline
\end{tabular}



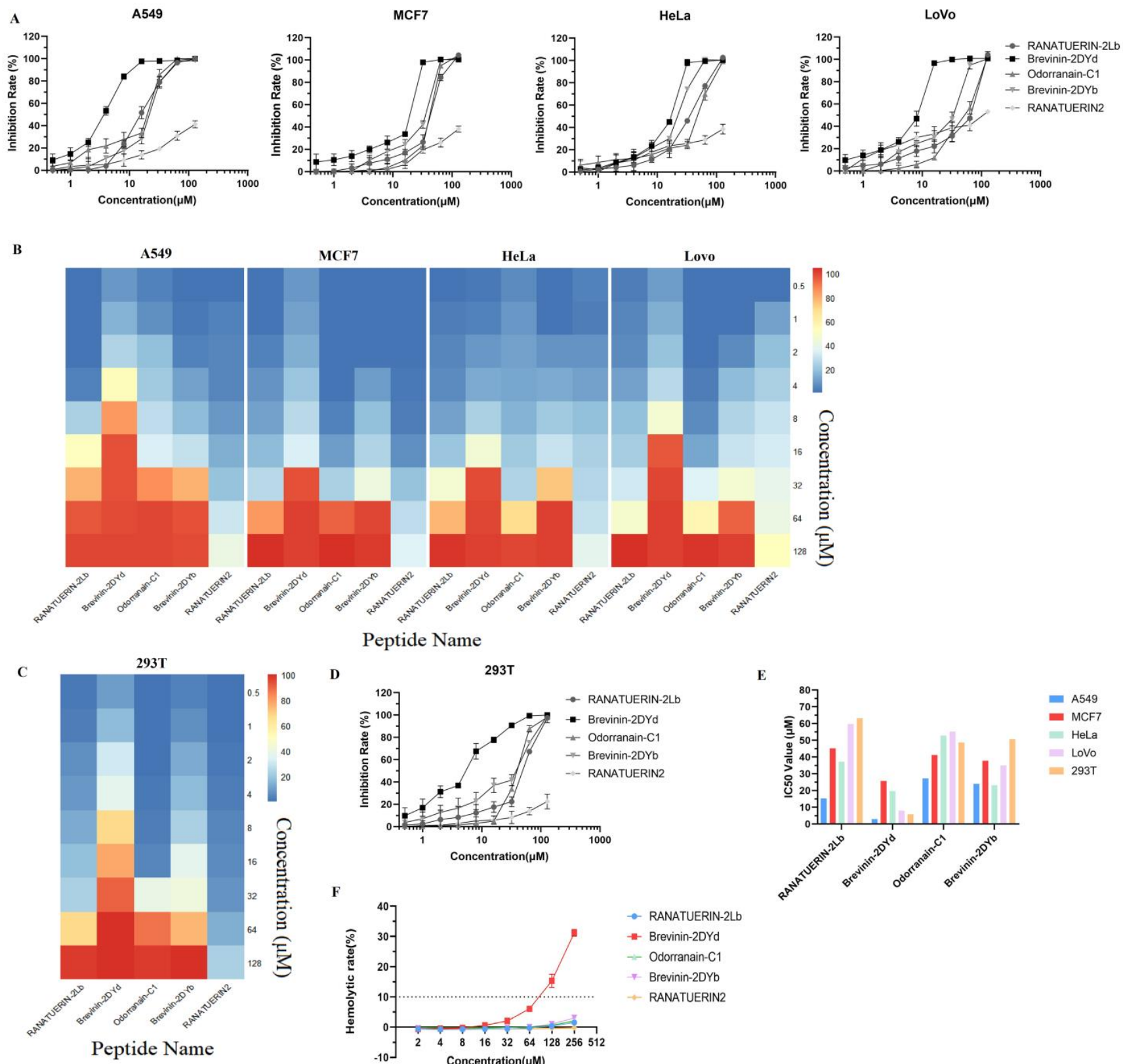

MCF7
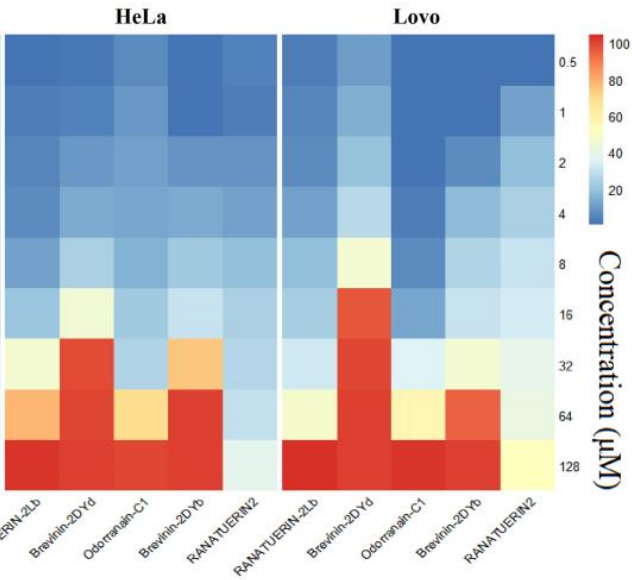

Peptide Name

D

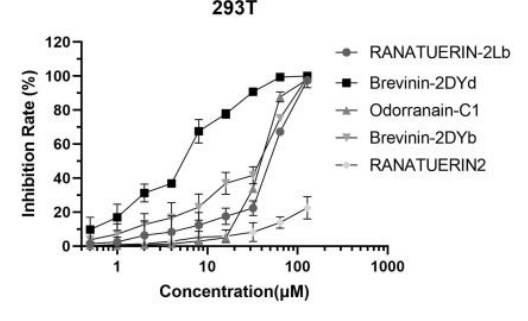

F

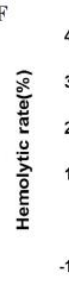

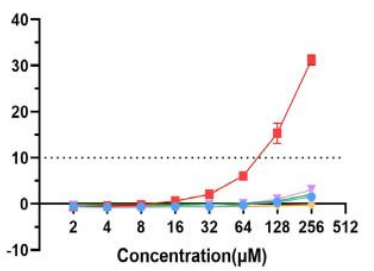

- RANATUERIN-2L

- Brevinin-2DYd

- Odorranain-C1

*- Brevinin-2DY

RANATUERIN2

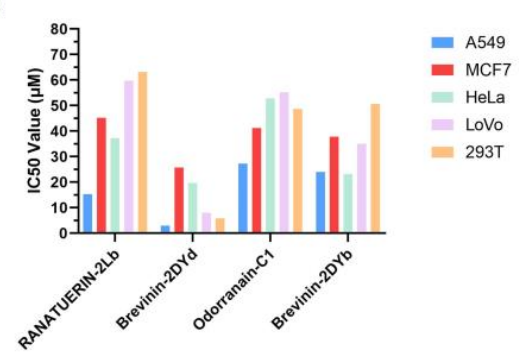

Figure 6. In vitro anticancer activity and toxicity of 5 peptides. (A) The inhibition rates of 5 peptides against 4 types of cancer cells (A549, MCF7, HeLa and LoVo) were calculated at different concentrations. (B) The heat map plot was used to show the inhibition rate of 5 peptides on diverse cancer cells. Inhibition rate is the average of three replicates. (C,D) Inhibition rate of human embryonic kidney cells 293T under 5 peptides concentrations. (E) The IC50 values of 4 peptides on 5 cell lines. (F) Hemolytic activities of selected ACPs at different concentrations.

To verify the effectiveness of the toxic peptide model, we selected human embryonic kidney cells 293T to test the inhibitory effect of candidate ACPs on non-cancerous cells (Figure 6C-E, Table 6). Similar to the division of ACPs activity, peptides were considered to have low toxicity when the IC50 value exceeded $50 \mu \mathrm{M}$. Three (RANATUERIN-2Lb, Brevinin-2DYb, RANATUERIN2) of the five peptides showed low toxicity to 293T cells, Odorranain-C1 exhibited certain toxicity and Brevinin-2DYd showed the highest toxicity. To a certain extent, the experimental results suggested that the prediction model of toxic peptide had a certain predictability, which was consistent with the model verification results.

We further reported the hemolytic activity of the 5 peptides, the hemolytic rate of $100 \mu \mathrm{M}$ peptides was more than $10 \%$ as the classification standard of hemolysis, and there 
was no hemolysis in the 4 peptides (RANATUERIN-2Lb, Odorranain-C1, Brevinin-2DYb and RANATUERIN2) and moderate hemolysis in Brevinin-2DYd (Figure 6F, Table 7). To some extent, these results indicate that the developed RF model derived from WEKA is effective in the prediction of hemolytic peptides.

Table 7. Hemolytic effect of 5 peptides on $2 \%$ sheep erythrocytes.

\begin{tabular}{|c|c|c|c|c|c|c|c|c|c|c|}
\hline \multirow[b]{2}{*}{ Peptides } & \multicolumn{10}{|c|}{ Peptide Concentration $(\mu \mathrm{M})$} \\
\hline & 2 & 4 & 8 & 16 & 32 & 64 & 128 & 256 & $\begin{array}{c}\text { Negative } \\
\text { Control } \\
\text { (PBS) }\end{array}$ & $\begin{array}{c}\text { Positive } \\
\text { Control (Triton } \\
\text { X-100 }(0.1 \%))\end{array}$ \\
\hline $\begin{array}{l}\text { RANATUERIN-2Lb } \\
(16563)\end{array}$ & - & - & - & - & - & - & - & - & - & + \\
\hline $\begin{array}{c}\text { Brevinin-2DYd } \\
(19566)\end{array}$ & - & - & - & - & - & - & + & + & - & + \\
\hline $\begin{array}{l}\text { Odorranain-C1 } \\
(22121)\end{array}$ & - & - & - & - & - & - & - & - & - & + \\
\hline $\begin{array}{c}\text { Brevinin-2DYb } \\
(27843)\end{array}$ & - & - & - & - & - & - & - & - & - & + \\
\hline $\begin{array}{l}\text { RANATUERIN2 } \\
(22355)\end{array}$ & - & - & - & - & - & - & - & - & - & + \\
\hline
\end{tabular}

The "+" symbol indicates that the hemolysis rate exceeds $10 \%$ when peptides concentration reaches $100 \mu \mathrm{M}$. The " - " symbol indicates the hemolysis rate of less than $10 \%$ at peptides concentration of $100 \mu \mathrm{M}$.

In summary, these results show that RANATUERIN-2Lb and Brevinin-2DYb are potential ACPs considering the anticancer activity and safety of peptides. RANATUERIN2Lb and Brevinin-2DYb have selective killing effect on lung cancer A549 cells relative to $293 \mathrm{~T}$ cells, and can be developed as potential candidate drugs for lung cancer. These experiments are only to validate the practicability of the developed ACPs, hemolytic peptides and cytotoxic peptides models, and more systematic experiments are needed to further develop ACP drugs.

\section{Discussion}

The purpose of this study was to construct models for predicting anticancer activity and safety of peptides based on their 3D structures, and to collect different datasets to compare the differences between the models developed by natural peptides and chemically modified peptides. In the ACPs datasets, $\mathrm{KNN}$ and RF of the classical algorithm are excellent, while AdaBoostM1, Bagging, Vote and Stacking of the ensemble algorithm show high accuracy. Compared with the previous model prediction methods for ACPs [10-26], features were extracted based on the 3D structure of peptides for the first time, which can be used as a supplementary method for the prediction of ACPs. The extraction of features from the 3D structure of peptides can better reflect the state of peptides in organisms and analyze the properties of peptides from different perspectives. Compared with the existing prediction models such as ENNAACT [26] and AntiCP 2.0 [23], the ACPs model developed by us is very robust only by its accuracy and MCC. However, due consideration has to be given to the differences caused by different methods of extracting features, so it is uncertain whether our model is superior to other models. The results of the test set of the model constructed in the ACPs dataset were slightly better than those of the training set, which may be due to the fact that the sample size of the test set is small, and the characteristics of positive and negative samples are clearly distinguished. Meanwhile, the ensemble algorithm adopted can prevent the overfitting of the training set, so as to maintain the good fit of the model and improve the external generalization ability as much as possible. Some peptide prediction models also show similar results, such as the model built by Piyush. et al., using the SVM algorithm [23], and the model developed by Vishuda. et al., using the RF algorithm [39].

Similarly, it is the first time to predict both hemolysis and toxicity of anticancer peptides while predicting their activities. Referred to the model developed by Vinod et al. using a similar approach [32], our model constructed in the hemolytic peptide chemical 
modification dataset HD2 achieved good results. However, our model in the toxic peptide dataset and the existing model uses different methods of feature extraction, so it is hard to compare the model performance. Multiple datasets collected in this study, the ACPs and hemolytic peptides collected were verified by experiments, while the data of toxic peptide datasets are all kinds of peptides with high toxicity, not just toxicity to non-cancerous cells. Toxic peptides act on non-cancer cells through a variety of mechanisms including interactions with specific ion channels, enzymes, mitochondria and membrane components. Therefore, the model for predicting hemolysis and toxicity developed in this study can be used to predict the toxic and side effects of various peptides. In order to further improve the accuracy of toxicity prediction, it is necessary to collect relatively appropriate datasets in the future.

In the classical peptide databases APD3 [40], $\mathrm{CAMP}_{\mathrm{R} 3}$ [41], DRAMP 2.0 [42] and SATPdb [34], the number of ACPs accounts for about $10 \%$ of AMPs. Thus, some AMPs exhibited anticancer activity [43], a candidate dataset containing 1294 AMPs was collected to screen novel ACPs. The hybrid ACPs model and the optimal hemolytic and toxicity prediction model developed in this study were applied to the candidate dataset to screen out 41 candidate ACPs and 5 of them were randomly selected for experimental verification. To a certain extent, the results of this study indicate that the mixed model for screening ACPs had excellent practicability, the hemolytic peptide model also had good applicability, and the toxic peptide model had a certain predictability. Taken together, these results suggest that RANATUERIN-2Lb and Brevinin-2DYb have both anticancer activity and safety, and are expected to be developed as candidate ACPs drugs. The method proposed in this study provides a new idea for predicting the activity and safety of ACPs. Although we obtained good results by selecting peptides with good predicted effects for experimental verification, and had a certain effect on predicting candidate ACPs drugs, the experimental verification method was not perfect enough from the perspective of model verification. As they have suggested, the peptides with the worst ensemble prediction results should be selected for further experiments to fully demonstrate the efficacy of the developed model.

Overall, we developed models for predicting the activity and safety of ACPs based on the 3D structure of peptides, and identified two novel candidate ACPs. Although the present method is restricted by the time consuming of the structure prediction step, it is still a powerful complement to the method of building models based on sequence features. With the development of computational biology, more and more 3D structure-based peptide activity prediction methods are expected to be developed and used widely. In order to verify whether the model is reliable, we randomly selected 5 peptides for experiment, and we will continue to conduct experiments on the remaining 36 candidate peptides to select the ACPs with high efficiency and low toxicity in the future. Considering that the structure of the peptide may change when it interacts with the cell, it is important to verify its three-dimensional structure through experiments. At present, the methods of protein crystal structure analysis mainly include nuclear magnetic resonance (NMR), X-ray diffraction imaging (XDI) and cryo-electron microscopy (Cryo-EM) [44]. Due to the limitation of experimental conditions, the 3D structure verification experiment of peptide cannot be carried out, so we hope to improve it in the subsequent studies. In this study, the selection of candidate ACPs mainly focused on natural ACPs, while the general chemically modified ACPs had better effects. Based on the excellent prediction model of chemically modified peptides developed in this paper, chemically modified peptides with high anticancer activity will be screened in future studies. 


\section{Materials and Methods}

\subsection{Datasets}

\subsubsection{ACPs Datasets}

In this study, we created 5 datasets in order to compare the differences between diverse datasets in constructing the prediction model of ACPs. We collected sequence and structure data on experimentally validated ACPs in CancerPPD [45] and SATPdb. Afterwards, natural peptides and chemically modified peptides are classified according to whether there are chemical modifications. Generally, acetylation, amidation, methylation, glycosylation and non-natural residues are counted as chemical modifications [46]. We extracted 435 natural ACPs from CancerPPD and SATPdb, and collected 300 chemically modified ACPs from CancerPPD. In order to remove sequence redundancy in the dataset, CD-Hit [47] was used to delete sequences with more than $85 \%$ similarity, and 280 natural ACPs were obtained. The non-anticancer AMPs were selected from SATPdb as nonACPs, 360 chemically modified non-ACPs were extracted and 471 natural non-ACPs were screened using CD-Hit with sequence identity cut-off of $85 \%$. In addition, we retrieved 356 random peptides from SwissProt [48] Proteins using the following keywords, "not anticancer activity", "amino acid length range of 5 to 70 " and "with 3D structure", as another natural non-ACPs dataset.

In order to create several balanced datasets, 280 natural ACPs and 280 natural AMPs from the SATPdb were selected as the D1 dataset. The D2 dataset was composed of 280 natural ACPs and 280 random peptides from SwissProt. The D3 dataset was made of 300 chemically modified ACPs and 300 chemically modified AMPs from SATPdb. Subsequently, natural peptides and chemically modified peptides were placed in one dataset to form a mixed dataset, that is, D1 and D3 constitute the mixed dataset D4, and D2 and D3 form the mixed dataset D5 (Figure 2A). The sequence and 3D structures data of the five ACP datasets were shown in Supplementary Materials 2.

\subsubsection{Hemolytic Peptides Datasets}

The sequence and structure data of hemolytic peptides verified by experiments were obtained from Hemolytik [49] and SATPdb, and natural peptides and chemically modified peptides were collected respectively. Peptides that satisfy one of the following criteria are considered hemolytic peptides, (i) minimum hemolytic concentration $(\mathrm{MHC}) \leq 250 \mu \mathrm{g} / \mathrm{mL}$; (ii) half maximum effective concentration (EC50) or hazardous concentration $(\mathrm{HC} 50) \leq 100 \mu \mathrm{M}$ and (iii) $>10 \%$ hemolytic activity up to $100 \mu \mathrm{M}$ [28]. Peptides that do not meet the above criteria are selected as non-hemolytic peptides with extremely low hemolysis at relatively high concentrations. Finally, the natural dataset HD1 of 94 hemolytic peptides and 94 non-hemolytic peptides was obtained through CD-Hit screening. Chemically modified peptide dataset HD2 was composed of 135 hemolytic peptides and 135 non-hemolytic peptides, and HD1 and HD2 constituted the mixed dataset HD3 (Figure 2B). Peptide sequences and 3D structures of the three hemolytic peptide datasets were shown in Supplementary Materials 2.

\subsubsection{Toxic Peptides Datasets}

Similarly, the structure and sequence data of toxic peptides were obtained from the SATPdb, which were divided into natural peptides and chemically modified peptides. The majority of toxic peptides in the SATPdb are peptide toxins from ATDB [50], Tox-Prot [51], ConoServer [52] and DBETH [53], which are usually highly toxic and have a killing effect on many types of cells, including non-cancer cells. Non-toxic AMPs were retrieved from SATPdb as non-toxic peptides, that is, negative datasets. We extracted the natural dataset TD1 of 1000 toxic peptides and 1000 non-toxic peptides by CD-Hit screening. The chemical modification dataset TD2 was formed from 120 toxic peptides and 120 non-toxic peptides, and TD1 and TD2 are mixed into the TD3 dataset (Figure 2C). Peptide sequences and 3D structures of the three toxic peptide datasets were shown in Supplementary Materials 2. 


\subsubsection{Candidate Datasets}

Since some AMPs also have anticancer properties, we collected a candidate dataset that did not include experimentally validated anticancer peptides in the hope of finding novel ACPs. A total of 2024 non-anticancer and non-toxic antibacterial peptides were obtained from the desired functions module of SATPdb. Then, CD-Hit was used to exclude peptides with sequences that were more than $90 \%$ similar to those in all study datasets. Finally, 1294 antibacterial peptides with predicted 3D structures were obtained to compose the candidate dataset.

\subsection{Internal and External Validations}

The length of amino acid sequences in all of the above datasets ranged from 5 to 70 . Each dataset was randomly divided into two datasets, that is, $80 \%$ of the data constituted the training set and the remaining $20 \%$ of the data constituted the test set, and the data ratio between positive samples and negative samples in each subset was about 1:1 (Figure 2D). The training set was used to train the model, and the 5 -fold cross validation technique was used for internal validation. For external validation, we used a test set to evaluate the performance of the trained model.

\subsection{Feature Extraction and Selection}

Based on the 3D structure of peptides, the global physicochemical descriptor of the 2018 MOE software [54] was used to extract the features related to the structural properties. The peptides of the same category usually have similar features and a prediction model can be built to distinguish them from other categories of peptides by using machine learning algorithms to recognize their common features. The 3D structure of peptides is closer to the real state of its action in living organisms, so the extracted features can better reflect the properties of peptide drugs. MOE descriptors contain $2062 \mathrm{D}$ descriptors, 148 3D descriptors and 88 protein descriptors, resulting in a total of 435 features. These features include volume and molecular shape, surface area, energy-related descriptors, conformation dependent charge descriptors, etc. To avoid data redundancy, descriptors with poor correlation with activity or toxicity should be removed for preliminary features screening. For every dataset, contingency coefficient C, Cramer's V, entropic uncertainty U and linear correlation $\mathrm{R}^{2}$ of each descriptor were calculated in MOE, and descriptors whose numerical value of $C, V, U$ and $R^{2}$ were 0 were deleted. The remaining descriptors are used for model construction. Then, in each dataset we used the WEKA package [55] for feature selection based on the above descriptors. We choose "CfsSubsetEval" as the evaluator and "Best First" as the default parameter of the search method, that is, the forward direction with amount of backtracking, $N=5$ and the lookup size $\mathrm{D}=1$ [46]. In addition, after the calculation of $\mathrm{C}, \mathrm{U}, \mathrm{V}$ and $\mathrm{R}^{2}$ mentioned above, MOE has recommended descriptors in some datasets and we built the model according to these descriptors.

\subsection{Structural Similarity Analysis}

A principal component analysis (PCA) was performed on the remaining descriptors of each dataset after preliminary feature screening. Then, the first three principal components were selected to perform the structural feature similarity analysis of $3 \mathrm{D}$ visualization for each dataset. The more concentrated the data distribution, the higher the similarity of peptide features.

\subsection{Machine Learning Techniques}

In this paper, we adopted a variety of machine learning algorithms to establish the model, which was mainly divided into two categories: classical algorithm and ensemble algorithm (Figure 1). Classical algorithms include Binary, KNN, RF and SVM. The Binary method is an algorithm based on Bayesian statistics, which can build classification models in the QSAR module of MOE software (2018), and uses the LOO (Leave one out) method for internal verification. The KNN algorithm was run in the Windows command window, 
the value range of $k$ is $1-9$. We used Euclidian distance to describe the similarity between samples, and the adjacent samples were classified into one category. Genetic algorithms with population size of 200 and termination algebra of 300 were used to screen models with correct classification rate (CCR) over 0.6. RF and SVM were implemented by using the random Forest and e1071 package in R respectively. RF uses a set of unpruned decision trees and randomly selects a subset of predictors as candidates for splitting tree nodes [56]. The SVM algorithm uses the radial basis kernel (RBK) function to construct the model, and sets two key parameters that cost as 1000 and gamma as $1 \times 10^{-6}$.

Ensemble algorithms mainly include AdaBoostM1 [57], Bagging [58], Stacking [59] and Vote [60] derived from WEKA software (version 3.8.4). AdaBoostM1 chose DecsionStump (DS) as the primary classifier, and DS, J48, SMO and NaiveBayes (NB) were selected respectively to build four algorithms. Bagging selected REPtree as the basic classifier, and REPtree, J48, SMO, NB, RF and IBK were selected to build six models. Stacking selects ZeroR, PART, OneR, J48, RF, IBK, NB and SMO as the basic learners and J48 as the secondary classifier to build an efficient model. Vote combines multiple algorithms and classifies samples according to the average probability of output. In order to obtain the model with excellent performance, the "CVParameterSelection" method was selected in WEKA to optimize the corresponding parameters of the algorithm.

\subsection{Performance Evaluation}

The performance of the evaluation model was represented by the following parameters: sensitivity (Sen), specificity (Spc), accuracy (Acc) and the Matthew correlation coefficient (MCC). The formula is shown below:

$$
\begin{gathered}
\text { Sen }=\frac{\mathrm{TP}}{\mathrm{TP}+\mathrm{FN}} \times 100 \% \\
\mathrm{Spc}=\frac{\mathrm{TN}}{\mathrm{TN}+\mathrm{FP}} \times 100 \% \\
\mathrm{Acc}=\frac{\mathrm{TP}+\mathrm{TN}}{\mathrm{TP}+\mathrm{FN}+\mathrm{TN}+\mathrm{FP}} \times 100 \% \\
\mathrm{MCC}=\frac{\mathrm{TP} \times \mathrm{TN}-\mathrm{FP} \times \mathrm{FN}}{\sqrt{(\mathrm{TP}+\mathrm{FP})(\mathrm{TP}+\mathrm{FN})(\mathrm{TN}+\mathrm{FP})(\mathrm{TN}+\mathrm{FN})}}
\end{gathered}
$$

where TP, FP, TN and FN stand for the number of true positives, false positives, true negatives and false negatives, respectively.

\subsection{Materials}

Five peptides were randomly selected from the candidate ACPs and synthesized by Changzhou Kanglong Biotech Ltd (Changzhou, China). using 9-fuorenylmethoxy carbonyl (FMOC) solid phase synthesis technology. The synthetic peptide was identified by mass spectrometry (MS), and the purity $>95 \%$ by high performance liquid chromatography (HPLC). Human breast cancer cell line (MCF7), lung cancer cell line (A549), cervical cancer cell line (HeLa), colorectal adenocarcinoma cell line (LoVo) and human embryonic kidney cells (293T) were preserved in our laboratory. At $37{ }^{\circ} \mathrm{C}$ under $5 / 95 \% \mathrm{CO}_{2} /$ air condition, all cell lines were cultured in Dulbecco's modified Eagle medium (DMEM) supplemented with 10\% ( $/ / v)$ fetal bovine serum (FBS, Gibco, Carlsbad, CA, USA) and 1\% (v/v) penicillin/streptomycin (Sangon Biotech, Shanghai, China).

\subsection{Cell Killing Ability Assay In Vitro}

Human tumor cell lines MCF7, A549, HeLa and LoVo were used as experimental materials to detect the anticancer activity of peptides by MTT assay [61,62]. In addition, human embryonic kidney cells 293T were also included in the experiment to examine the safety of the peptide against non-cancerous cells. First, the cells were cultured, then the cells were inoculated in 96-well plates (6000 cells per well) and incubated for $24 \mathrm{~h}$, 
followed by the addition of five peptides $(0.5,1,2,4,8,16,32,64$ and $128 \mu \mathrm{M})$ at increasing concentrations for $24 \mathrm{~h}$, each concentration was prepared in triplicate. Untreated cells as negative control group incubated with corresponding medium for $24 \mathrm{~h}$. Subsequently, $10 \mathrm{uL}, 5 \mathrm{mg} / \mathrm{mL}$ MTT (Sangon Biotech, Shanghai, China) solution was added to each well and incubated for $4 \mathrm{~h}$ at $37^{\circ} \mathrm{C}$. After the medium was discarded, $150 \mu \mathrm{L}$ DMSO was added to each well for $10 \mathrm{~min}$ to dissolve formazan crystals. Absorbance was measured at 570 and $630 \mathrm{~nm}$ using a multiwall plate reader and the inhibition rate was expressed as the mean \pm standard deviation (SD) of the triplicate data.

\subsection{Hemolysis Assay}

The $2 \%$ sheep red blood cells (SRBC) were purchased from Nanjing Senbeijia Biological Technology Co., Ltd (Nangjing, China). A total of $4 \mathrm{~mL}$ of $2 \%$ SRBC was taken, rinsed twice with $4 \mathrm{~mL}$ of PBS by centrifugation for $5 \mathrm{~min}$ at $3000 \mathrm{rpm}$ and the precipitates were resuspended in $4 \mathrm{~mL}$ of PBS. We added $100 \mu \mathrm{L}$ of $2 \%$ SRBC to each well of the 96-well plate and then added $100 \mu \mathrm{L}$ peptide solutions of different concentrations $(2,4,8,16,32,64$, 128 and $256 \mu \mathrm{M}$ ) in triplicate at $37^{\circ} \mathrm{C}$ for $1 \mathrm{~h}$. $100 \mu \mathrm{L}$ of PBS buffer and $100 \mathrm{uL}$ of Triton X-100 0.1\% (w/v) were mixed with SRBC as negative and positive controls [63], respectively. All samples were centrifuged at $3000 \mathrm{rpm}$ for $5 \mathrm{~min}, 100 \mu \mathrm{L}$ supernatant was collected and transferred to a new 96-well plate, and its absorbance was measured at $540 \mathrm{~nm}$. The calculation formula of hemolysis rate is as follows.

$$
\% \text { Hemolysis }=\frac{\text { absorbance }_{\text {sample }}-\text { absorbance }_{\text {negative }}}{\text { absorbance }_{\text {positive }}-\text { absorbance }_{\text {negative }}} \times 100
$$

Supplementary Materials: The following are available online at https:/ / www.mdpi.com/article/10 $.3390 /$ ijms22115630/s1.

Author Contributions: Conceptualization, M.W. (Min Wu), M.W. (Min Wang) and Y.Z.; methodology, M.W. (Min Wu) and Y.Z.; Data collection and algorithm validation, Y.Z. and S.W.; experimental verification, Y.Z., S.W., W.F., Y.F., L.S. and X.Y.; writing-original draft preparation, Y.Z.; writingreview and editing, M.W. (Min Wu) and M.W. (Min Wang); supervision, M.W. (Min Wu) and M.W. (Min Wang). All authors have read and agreed to the published version of the manuscript.

Funding: This work was supported by funds from the National Natural Science Foundation of China (NSFC81703404), a project funded by the Priority Academic Program Development of Jiangsu Higher Education Institutions (PAPD), Double First-Class University Project (CPU2018GY14), a project fund by the Graduate Innovation Program of Jiangsu Province (SJKY19_0695) and College Students Innovation Project for the R\&D of Novel Drugs.

Institutional Review Board Statement: Not applicable.

Informed Consent Statement: Not applicable.

Data Availability Statement: The data discussed in this publication can be obtained in Supplementary Materials 2 (https: / / www.mdpi.com/article/10.3390/ijms22115630/s1).

Conflicts of Interest: The authors declare no conflict of interest.

\section{References}

1. Sung, H.; Ferlay, J.; Siegel, R.L.; Laversanne, M.; Soerjomataram, I.; Jemal, A.; Bray, F. Global cancer statistics 2020: GLOBOCAN estimates of incidence and mortality worldwide for 36 cancers in 185 countries. CA Cancer J. Clin. 2021, 70, 1-41. [CrossRef]

2. Siegel, R.L.; Miller, K.D.; Goding Sauer, A.; Fedewa, S.A.; Butterly, L.F.; Anderson, J.C.; Cercek, A.; Smith, R.A.; Jemal, A. Colorectal cancer statistics, 2020. CA Cancer J. Clin. 2020, 70, 145-164. [CrossRef]

3. Liscano, Y.; Oñate-Garzón, J.; Delgado, J.P. Peptides with dual antimicrobial-anticancer activity: Strategies to overcome peptide limitations and rational design of anticancer peptides. Molecules 2020, 25, 4245. [CrossRef]

4. Craik, D.J.; Fairlie, D.P.; Liras, S.; Price, D. The future of peptide-based drugs. Chem. Biol. Drug Des. 2013, 81, 136-147. [CrossRef] [PubMed]

5. Felício, M.R.; Silva, O.N.; Gonçalves, S.; Santos, N.C.; Franco, O.L. Peptides with dual antimicrobial and anticancer activities. Front. Chem. 2017, 5, 1-9. [CrossRef] 
6. Chen, F.; Ma, B.; Yang, Z.C.; Lin, G.; Yang, D. Extraordinary metabolic stability of peptides containing $\alpha$-aminoxy acids. Amino Acids 2012, 43, 499-503. [CrossRef]

7. Shoombuatong, W.; Schaduangrat, N.; Nantasenamat, C. Unraveling the bioactivity of anticancer peptides as deduced from machine learning. EXCLI J. 2018, 17, 734-752. [CrossRef]

8. Tyagi, A.; Kapoor, P.; Kumar, R.; Chaudhary, K.; Gautam, A.; Raghava, G.P.S. In silico models for designing and discovering novel anticancer peptides. Sci. Rep. 2013, 3, 1-8. [CrossRef]

9. Hajisharifi, Z.; Piryaiee, M.; Mohammad Beigi, M.; Behbahani, M.; Mohabatkar, H. Predicting anticancer peptides with Chou's pseudo amino acid composition and investigating their mutagenicity via Ames test. J. Theor. Biol. 2014, 341, 34-40. [CrossRef]

10. Saravanan, V.; Lakshmi, P.T.V. ACPP: A web server for prediction and design of anti-cancer peptides. Int. J. Pept. Res. Ther. 2015, 21, 99-106. [CrossRef]

11. Chen, W.; Ding, H.; Feng, P.; Lin, H.; Chou, K.C. iACP: A sequence-based tool for identifying anticancer peptides. Oncotarget 2016, 7, 16895-16909. [CrossRef]

12. Manavalan, B.; Basith, S.; Shin, T.H.; Choi, S.; Kim, M.O.; Lee, G. MLACP: Machine-learning-based prediction of anticancer peptides. Oncotarget 2017, 8, 77121-77136. [CrossRef]

13. Akbar, S.; Hayat, M.; Iqbal, M.; Jan, M.A. iACP-GAEnsC: Evolutionary genetic algorithm based ensemble classification of anticancer peptides by utilizing hybrid feature space. Artif. Intell. Med. 2017, 79, 62-70. [CrossRef] [PubMed]

14. Wei, L.; Zhou, C.; Chen, H.; Song, J.; Su, R. ACPred-FL: A sequence-based predictor using effective feature representation to improve the prediction of anti-cancer peptides. Bioinformatics 2018, 34, 4007-4016. [CrossRef]

15. Xu, L.; Liang, G.; Wang, L.; Liao, C. A novel hybrid sequence-based model for identifying anticancer peptides. Genes 2018, 9, 158. [CrossRef] [PubMed]

16. Kabir, M.; Arif, M.; Ahmad, S.; Ali, Z.; Swati, Z.N.K.; Yu, D.J. Intelligent computational method for discrimination of anticancer peptides by incorporating sequential and evolutionary profiles information. Chemom. Intell. Lab. Syst. 2018, 182, 158-165. [CrossRef]

17. Schaduangrat, N.; Nantasenamat, C.; Prachayasittikul, V.; Shoombuatong, W. ACPred: A computational tool for the prediction and analysis of anticancer peptides. Molecules 2019, 24, 1973. [CrossRef]

18. Boopathi, V.; Subramaniyam, S.; Malik, A.; Lee, G.; Manavalan, B.; Yang, D.C. MACppred: A support vector machine-based meta-predictor for identification of anticancer peptides. Int. J. Mol. Sci. 2019, 20, 1964. [CrossRef]

19. Rao, B.; Zhou, C.; Zhang, G.; Su, R.; Wei, L. ACPred-Fuse: Fusing multi-view information improves the prediction of anticancer peptides. Brief. Bioinform. 2019, 21, 1-10. [CrossRef]

20. Wu, C.; Gao, R.; Zhang, Y.; De Marinis, Y. PTPD: Predicting therapeutic peptides by deep learning and word2vec. BMC Bioinform. 2019, 20, 456. [CrossRef]

21. Yi, H.C.; You, Z.H.; Zhou, X.; Cheng, L.; Li, X.; Jiang, T.H.; Chen, Z.H. ACP-DL: A deep learning long short-term memory model to predict anticancer peptides using high-efficiency feature representation. Mol. Ther. Nucleic Acids 2019, 17, 1-9. [CrossRef]

22. Wei, L.; Zhou, C.; Su, R.; Zou, Q. PEPred-Suite: Improved and robust prediction of therapeutic peptides using adaptive feature representation learning. Bioinformatics 2019, 35, 4272-4280. [CrossRef]

23. Agrawal, P.; Bhagat, D.; Mahalwal, M.; Sharma, N.; Raghava, G.P.S. AntiCP 2.0: An updated model for predicting anticancer peptides. Brief. Bioinform. 2020, 22, bbaa153. [CrossRef]

24. Burdukiewicz, M.; Sidorczuk, K.; Rafacz, D.; Pietluch, F.; Bąkała, M.; Słowik, J.; Gagat, P. Cancergram: An effective classifier for differentiating anticancer from antimicrobial peptides. Pharmaceutics 2020, 12, 1045. [CrossRef]

25. Yu, L.; Jing, R.; Liu, F.; Luo, J.; Li, Y. DeepACP: A Novel Computational Approach for Accurate Identification of Anticancer Peptides by Deep Learning Algorithm. Mol. Ther. Nucleic Acids 2020, 22, 862-870. [CrossRef]

26. Timmons, P.B.; Hewage, C.M. ENNAACT is a novel tool which employs neural networks for anticancer activity classification for therapeutic peptides. Biomed. Pharmacother. 2021, 133, 111051. [CrossRef] [PubMed]

27. Gupta, S.; Kapoor, P.; Chaudhary, K.; Gautam, A.; Kumar, R.; Raghava, G.P.S. In silico approach for predicting toxicity of peptides and proteins. PLoS ONE 2013, 8, e73957. [CrossRef]

28. Chaudhary, K.; Kumar, R.; Singh, S.; Tuknait, A.; Gautam, A.; Mathur, D.; Anand, P.; Varshney, G.C.; Raghava, G.P.S. A web server and mobile app for computing hemolytic potency of peptides. Sci. Rep. 2016, 6, 22843. [CrossRef]

29. Win, T.S.; Malik, A.A.; Prachayasittiku, V.; Wikberg, J.E.S.; Nantasenamat, C.; Shoombuatong, W. HemoPred: A web server for predicting the hemolytic activity of peptides. Future Med. Chem. 2017, 9, 275-291. [CrossRef] [PubMed]

30. Timmons, P.B.; Hewage, C.M. HAPPENN is a novel tool for hemolytic activity prediction for therapeutic peptides which employs neural networks. Sci. Rep. 2020, 10, 10869. [CrossRef]

31. Plisson, F.; Ramírez-Sánchez, O.; Martínez-Hernández, C. Machine learning-guided discovery and design of non-hemolytic peptides. Sci. Rep. 2020, 10, 16581. [CrossRef]

32. Kumar, V.; Kumar, R.; Agrawal, P.; Patiyal, S.; Raghava, G.P.S. A method for predicting hemolytic potency of chemically modified peptides from its structure. Front. Pharmacol. 2020, 11, 54. [CrossRef] [PubMed]

33. Mehedi, H.; Nalini, S.; Shaherin, B.; Gwang, L.; Watshara, S.; Balachandran, M. HLPpred-Fuse: Improved and robust prediction of hemolytic peptide and its activity by fusing multiple feature representation. Bioinformatics 2020, 36, 3350-3356. [CrossRef]

34. Singh, S.; Chaudhary, K.; Dhanda, S.K.; Bhalla, S.; Usmani, S.; Gautam, A.; Tuknait, A.; Agrawal, P.; Mathur, D.; Raghava, G.P.S. SATPdb: A database of structurally annotated therapeutic peptides. Nucleic Acids Res. 2016, 44, 1119-1126. [CrossRef] 
35. Singh, S.; Singh, H.; Tuknait, A.; Chaudhary, K.; Singh, B.; Kumaran, S.; Raghava, G.P.S. PEPstrMOD: Structure prediction of peptides containing natural, non-natural and modified residues. Biol. Direct 2015, 1, 73. [CrossRef] [PubMed]

36. Rodriguez, R.; Chinea, G.; Lopez, N.; Pons, T.; Vriend, G. Homology modeling, model and software evaluation: Three related resources. Bioinformatics 1998, 14, 523-528. [CrossRef]

37. Yang, J.; Yan, R.; Roy, A.; Xu, D.; Poisson, J.; Zhang, Y. The I-TASSER suite: Protein structure and function prediction. Nat. Methods 2014, 12, 7-8. [CrossRef]

38. Grisoni, F.; Neuhaus, C.S.; Hishinuma, M.; Gabernet, G.; Hiss, J.A.; Kotera, M.; Schneider, G. De novo design of anticancer peptides by ensemble artificial neural networks. J. Mol. Model. 2019, 25, 112. [CrossRef]

39. Laengsri, V.; Nantasenamat, C.; Schaduangrat, N.; Nuchnoi, P.; Prachayasittikul, V.; Shoombuatong, W. Targetantiangio: A sequence-based tool for the prediction and analysis of anti-angiogenic peptides. Int. J. Mol. Sci. 2019, 20, 2950. [CrossRef] [PubMed]

40. Wang, G.; Li, X.; Wang, Z. APD3: The antimicrobial peptide database as a tool for research and education. Nucleic Acids Res. 2016, 44, 1087-1093. [CrossRef]

41. Waghu, F.H.; Barai, R.S.; Gurung, P.; Idicula-Thomas, S. CAMPR3: A database on sequences, structures and signatures of antimicrobial peptides. Nucleic Acids Res. 2016, 44, 1094-1097. [CrossRef]

42. Kang, X.; Dong, F.; Shi, C.; Liu, S.; Sun, J.; Chen, J.; Li, H.; Xu, H.; Lao, X.; Zheng, H. DRAMP 2.0, an updated data repository of antimicrobial peptides. Sci. Data 2019, 6, 180258. [CrossRef]

43. Gaspar, D.; Freire, J.M.; Pacheco, T.R.; Barata, J.T.; Castanho, M.A.R.B. Apoptotic human neutrophil peptide-1 anti-tumor activity revealed by cellular biomechanics. Biochim. Biophys. Acta Mol. Cell Res. 2015, 1853, 308-316. [CrossRef]

44. Gurrola, G.B.; Guijarro, J.I.; Delepierre, M.; Mendoza, R.L.L.; Cid-Uribe, J.I.; Coronas, F.V.; Possani, L.D. Cn29, a novel orphan peptide found in the venom of the scorpion Centruroides noxius: Structure and function. Toxicon 2019, 167, 184-191. [CrossRef]

45. Tyagi, A.; Tuknait, A.; Anand, P.; Gupta, S.; Sharma, M.; Mathur, D.; Joshi, A.; Singh, S.; Gautam, A.; Raghava, G.P.S. CancerPPD: A database of anticancer peptides and proteins. Nucleic Acids Res. 2015, 43, 837-843. [CrossRef]

46. Agrawal, P.; Raghava, G.P.S. Prediction of antimicrobial potential of a chemically modified peptide from its tertiary structure. Front. Microbiol. 2018, 9, 2551. [CrossRef] [PubMed]

47. Li, W.; Godzik, A. Cd-hit: A fast program for clustering and comparing large sets of protein or nucleotide sequences. Bioinformatics 2006, 22, 1658-1659. [CrossRef]

48. Bateman, A.; Martin, M.J.; O’Donovan, C.; Magrane, M.; Apweiler, R.; Alpi, E.; Antunes, R.; Arganiska, J.; Bely, B.; Bingley, M.; et al. UniProt: A hub for protein information. Nucleic Acids Res. 2015, 43, 204-212. [CrossRef]

49. Gautam, A.; Chaudhary, K.; Singh, S.; Joshi, A.; Anand, P.; Tuknait, A.; Mathur, D.; Varshney, G.C.; Raghava, G.P.S. Hemolytik: A database of experimentally determined hemolytic and non-hemolytic peptides. Nucleic Acids Res. 2014, 42, 444-449. [CrossRef]

50. He, Q.Y.; He, Q.Z.; Deng, X.C.; Yao, L.; Meng, E.; Liu, Z.H.; Liang, S.P. ATDB: A uni-database platform for animal toxins. Nucleic Acids Res. 2008, 36, 293-297. [CrossRef]

51. Jungo, F.; Bairoch, A. Tox-Prot, the toxin protein annotation program of the Swiss-Prot protein knowledgebase. Toxicon 2005, 45, 293-301. [CrossRef]

52. Kaas, Q.; Westermann, J.C.; Halai, R.; Wang, C.K.L.; Craik, D.J. ConoServer, a database for conopeptide sequences and structures. Bioinformatics 2008, 24, 445-446. [CrossRef]

53. Chakraborty, A.; Ghosh, S.; Chowdhary, G.; Maulik, U.; Chakrabarti, S. DBETH: A database of bacterial exotoxins for human. Nucleic Acids Res. 2012, 40, 615-620. [CrossRef]

54. Molecular Operating Environment (MOE). Chemical Computing Group: Montreal, QC, Canada, 2018. Available online: https: //www.chemcomp.com (accessed on 10 December 2019).

55. Data Mining: Practical Machine Learning Tools and Techniques. 2018. Available online: https://www.cs.waikato.ac.nz/ml/ weka/book.html (accessed on 20 April 2020).

56. Liu, S.; Bao, J.; Lao, X.; Zheng, H. Novel 3d structure based model for activity prediction and design of antimicrobial peptides. Sci. Rep. 2018, 8, 11189. [CrossRef]

57. Schapire, R.E.; Singer, Y. Improved boosting algorithms using confidence-rated predictions. Mach. Learn. 1999, 37, 297-336. [CrossRef]

58. Breiman, L. Bagging predictors. Mach. Learn. 1996, 24, 123-140. [CrossRef]

59. Wolpert, D.H. Stacked generalization. Neural Netw. 1992, 5, 241-259. [CrossRef]

60. Kittler, J.; Hatef, M.; Duin, R.; Matas, J. On combining classifiers. IEEE Trans. Pattern Anal. Mach. Intell. 1998, 20, 226-239. [CrossRef]

61. Gholibeikian, M.; Bamoniri, A.; HoushdarTehrani, M.H.; Fatemeh Mirjalili, B.B.; Bijanzadeh, H.R. Structure-activity relationship studies of Longicalcynin A analogues, as anticancer cyclopeptides. Chem. Biol. Interact. 2020, 315, 108902. [CrossRef]

62. Wang, S.; Zhou, R.; Sun, F.; Li, R.; Wang, M.; Wu, M. The two novel DLL4-targeting antibody-drug conjugates MvM03 and MGD03 show potent anti-tumour activity in breast cancer xenograft models. Cancer Lett. 2017, 409, 125-136. [CrossRef]

63. Zhao, J.; Zhao, C.; Liang, G.; Zhang, M.; Zheng, J. Engineering antimicrobial peptides with improved antimicrobial and hemolytic activities. J. Chem. Inf. Model. 2013, 53, 3280-3296. [CrossRef] [PubMed] 